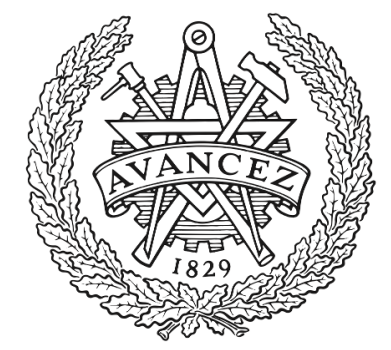

CHALMERS

UNIVERSITY OF TECHNOLOGY

\title{
Experimental and numerical investigations of cooling drag
}

Downloaded from: https://research.chalmers.se, 2023-04-26 11:47 UTC

Citation for the original published paper (version of record):

Hobeika, T., Sebben, S., Löfdahl, L. (2017). Experimental and numerical investigations of cooling drag. Proceedings of the Institution of Mechanical Engineers, Part D: Journal of Automobile Engineering, 231(9 (SI)): 1203-1210. http://dx.doi.org/10.1177/0954407016684740

N.B. When citing this work, cite the original published paper. 


\section{Experimental and Numerical Investigations of Cooling Drag}

\author{
Teddy Hobeika ${ }^{1}$, Simone Sebben ${ }^{1}$ and Lennart Löfdahl ${ }^{1}$
}

Journal Title

$\mathrm{XX}(\mathrm{X}): 1-10$

(C)The Author(s) 2016

Reprints and permission:

sagepub.co.uk/journalsPermissions.nav

DOI: 10.1177/ToBeAssigned

www.sagepub.com/

@SAGE

\begin{abstract}
As the target figures for $\mathrm{CO}_{2}$ emissions are reduced every year, vehicle manufacturers seek to exploit all possible gains in the different vehicle attributes. Aerodynamic drag is an important factor that affects the vehicles fuel consumption, and its importance rises with the shift from the New European Driving Cycle to the Worldwide harmonized Light vehicles Test Cycle which has a higher average speed. In order to reduce vehicle drag, car manufacturers employ the use of grill/spoiler shutters which reduces the amount of air going through the vehicles cooling system, also known as cooling flow, thus reducing both its cooling capability and the resultant cooling drag. This paper investigates the influence of different grill blockages on the cooling flow through the radiator of a Volvo S60. By modifying the engine bay and radiator, load cells are used to measure the force acting on the radiator core while the velocity distribution across the radiator core is measured using pressure probes. These values are analyzed and compared to different vehicle configurations and grill inlet designs. A number of test configurations are reproduced in Computational Fluid Dynamics simulations and compared to the test results. For some grill configurations, the simulations provide good prediction of mass flow and velocity distribution however a clear discrepancy is present as the grill blockages increase. On the other hand, the force acting on the radiator core was well predicted for all configurations. This paper discusses the different parameters affecting cooling flow predictions such as wind tunnel blockage and measurement grid discretization by comparing radiator forces and mass flows. In addition, the changes on overall vehicle forces are discussed with the radiator force put in context with cooling drag.
\end{abstract}

\title{
Keywords
}

Cooling flow, Cooling drag, CFD, vehicle cooling, vehicle aerodynamics

\section{Introduction}

As the target figures for $\mathrm{CO}_{2}$ emissions are reduced every year, vehicle manufacturers seek to exploit all possible gains in the different vehicle attributes. Aerodynamic drag is an important factor that affects the vehicles fuel consumption, and its importance rises with the shift from the New European Driving Cycle (NEDC) to the Worldwide harmonized Light vehicles Test Cycle (WLTC) which has a higher average speed. In order to reduce vehicle drag, car manufacturers employ the use of grill/spoiler shutters which reduces the amount of air going through the vehicles cooling system, also known as cooling flow, thus reducing both its cooling capability and the resultant cooling drag. Moreover, the introduction of grill shutters opens up many possibilities for optimizing the cooling flow, especially on hybrid electric vehicles and fully electrical vehicles. Even more control over cooling efficiency can be

\footnotetext{
${ }^{1}$ Chalmers University of Technology, Gothenburg, Sweden

Corresponding author:

Lennart Löfdahl, Applied Mechanics, Chalmers university of Technology, Hörsalsvägen 7A, Gothenburg, 41296, Sweden

Email: lennart.lofdahl@chalmers.se
} 
obtained by partly opening grill shutters or creating new packaging scenarios, yet the increase of uncertainties in optimization parameters is a challenge, both experimentally and numerically.

It is important to be able to predict the drag and lift changes due to different grill configurations in a constant optimization cycle between reducing drag and having sufficient cooling flow. With different alterations to grill openings it is difficult to isolate the increase in drag due to cooling flow from the interference effects this flow has with other vehicle geometry. Thus the term cooling drag emerges which is in practice ${ }^{1}$ the difference in drag between completely blocked cooling inlets and open inlet for the different grill configuration. Depending on the analysis being performed, there are many formulations used for cooling drag in both simple and complex forms ${ }^{2-5}$.

Garrone $^{6}$ has performed an experimental investigation where the drag on the cooling package is measured separately using load cells then compared to cooling drag. In his comparison the cooling package drag contribution of 17 count $\left(0.017 \mathrm{C}_{\mathrm{d}}\right)$ was significantly larger that the 3 count cooling drag measured on the vehicle. As the cooling package inlets and outlets are both ducted then the cooling package contribution is mostly attributed to the pressure loss across the radiator; by placing the cooling outlets in the low pressure wheelhouses a low cooling drag was achieved. Also for even more complex vehicles, Williams ${ }^{7}$ has shown that the losses in the engine bay are minor compared to losses from the cooling package.

Although challenging to separate the force acting on the radiator and the cooling drag in wind tunnel tests, it is quite easy in Computational Fluid Dynamics (CFD) simulations. In previous works, simulations have been able to predict general trends in overall vehicle forces yet the prediction of magnitudes remains challenging ${ }^{8}$. The cooling drag measured in wind tunnels is still influenced by blockage effects, and when specific quantities like radiator forces are being measured a correction could be needed as the blockage could affect the mass flow in the radiator ${ }^{9}$. Also, discrete pressure based mass flow measurements could introduce errors to cooling flow quantification due to the complexity of the flow and the measurement grid density ${ }^{10}$. For the mentioned reasons, this work quantifies the pressure loss through the radiator core by means of radiator force and mass flow measurements under the effect of large grill blockages, with the primary goal of comparing to CFD simulations. Effects of wind tunnel blockage and measurement grid discretization are discussed from both experimental and numerical perspective. Also a cooling flow indifference to wheel rotation, moving ground, and mesh refinement is presented. Finally, the changes in overall vehicle forces due to cooling flow are discussed and the radiator force is put in perspective with cooling drag.

\section{Methodology}

Full scale wind tunnel experiments and numerical simulations have been performed on a Volvo S60 Y283 model. Flow through the radiator, force acting on the radiator core, as well as overall vehicle forces are measured and analyzed. As the radiator flow and force are the main aim of this investigation, the geometry around it has been replicated with high accuracy in simulations to match the test object.

\section{Experimental Setup}

The wind tunnel tests are performed at the Volvo Cars wind tunnel, which is a closed loop wind tunnel with a slotted wall test section and equipped with a five belt moving ground system ${ }^{11}$. The tests reported in this paper are performed at $100 \mathrm{~km} / \mathrm{h}$ with an active moving ground system and rotating wheels, unless otherwise specified. The car engine bay has been modified for this particular test; most components of the cooling system have been removed with only the radiator and the fan shroud remaining. By doing so, the forces acting on the radiator core can be measured using a simple setup with two load cells on each side of the radiator. In order to eliminate interference from oncoming flow and to make it more representable of the normal vehicle conditions, special aluminum ducts guide the air from the bumper inlets straight to the radiator core thus shielding off the water tanks. With this setup six different grill configurations have been tested to evaluate the effect of grill blockage ranging from completely open to completely closed as presented in Figure 1. These grill blockages only cover the grill opening while the 


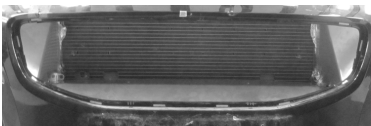

(a) Grill 1: Open grill

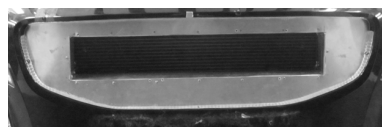

(c) Grill 3

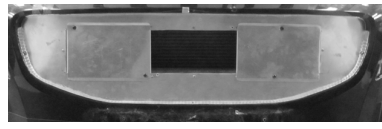

(e) Grill 5

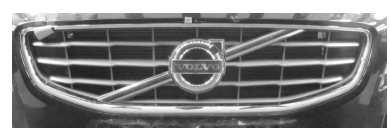

(b) Grill 2: Production grill

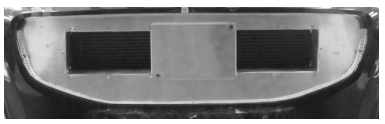

(d) Grill 4

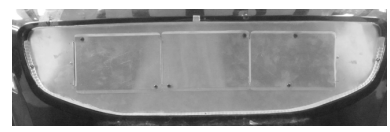

(f) Grill 6: Closed grill

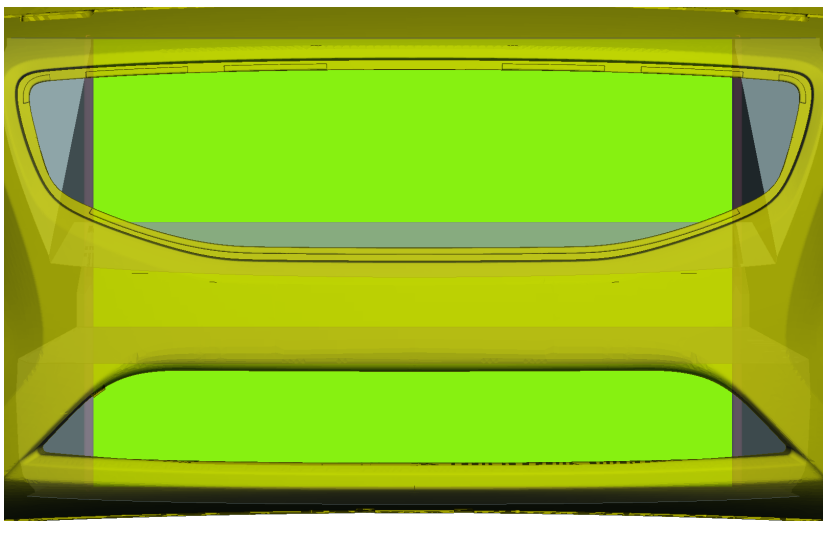

(a) Front blockage: bumper and aluminum ducts

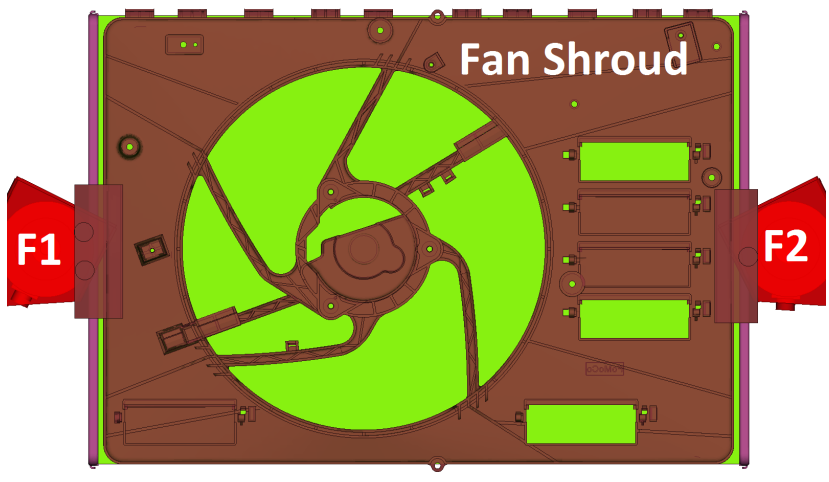

(b) Rear blockage: fan shroud and load cells (F1 and F2)

Figure 2. Figure showing radiator blockage from front and rear.

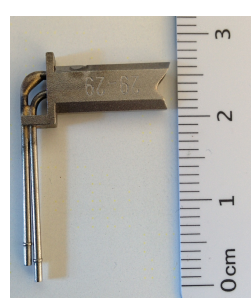

(a) Ruijsink Probe

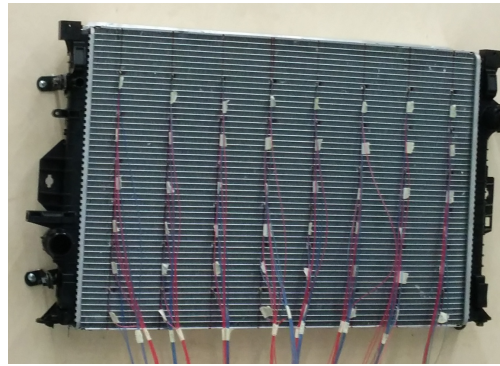

(b) Probe arrangement in the radiator
Figure 3. Geometrical setup of the 48 probes. low and high velocities, however at speeds below $2 \mathrm{~m} / \mathrm{s}$ the corrections vary largely with very small changes in velocity. From these 48 measurement points the velocity distribution across the radiator can be plotted, using linear interpolation or extrapolation, and the average radiator core velocity can be determined. However, as all pressure measurement techniques have limitations at low velocities where the viscous effects are strongly pronounced and they affect the measurement accuracy ${ }^{14}$, all velocities below $2 \mathrm{~m} / \mathrm{s}$ are unreliable $^{10}$.

\section{Numerical Setup}

The process for CFD simulations is carried out using commercial software: Ansa, for CAD cleaning, Harpoon, for meshing, and Fluent for solving. Much care was the geometry in the vicinity of the radiator. The front of the vehicle, aluminum ducts, and known flow outlets are dedicated to ensure that the numerical model replicates 


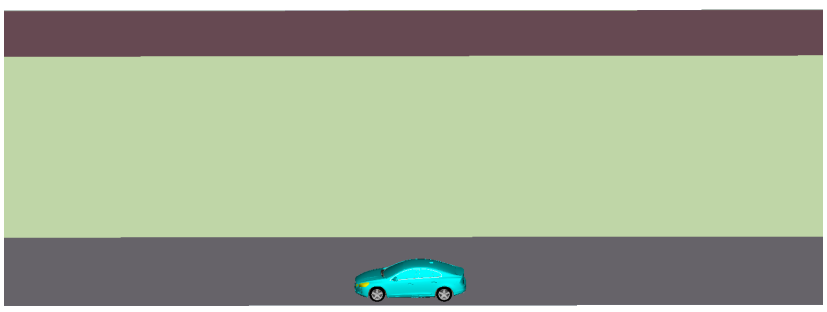

(a) Open road

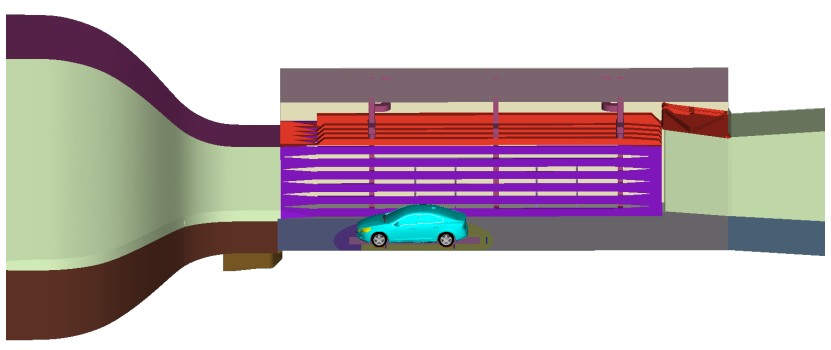

(b) Virtual tunnel

Figure 4. The test vehicle in: (a) open road, (b) virtual wind tunnel conditions.

resolved down to $2.5 \mathrm{~mm}$ while the radiator, load cells, fan shroud, and potential leakage areas are resolved down to $1.25 \mathrm{~mm}$. The remaining of the vehicle refinements vary from 1.25 to $5 \mathrm{~mm}$ following general external aerodynamics guidelines. The simulations have all been performed in open road conditions as well as in a virtual model of the Volvo wind tunnel ${ }^{15}$. The flow inlet and outlet boundary conditions in the wind tunnel are tuned in order to replicate the reference pressure drops measured by two probes located in the nozzle. This setup led to two sets of simulations, in open road and virtual tunnel, with 190 and 300 million cells respectively, shown in Figure 4. The simulations are solved with the k-epsilon turbulence model, enhanced-wall treatment, Green-Gauss node-based gradient scheme, and second order convective discretization schemes for pressure, momentum, turbulent dissipation rate, and turbulent kinetic energy. They are performed for a vehicle velocity of $100 \mathrm{~km} / \mathrm{h}$ with Moving Reference Frame (MRF) for modelling rim rotation.

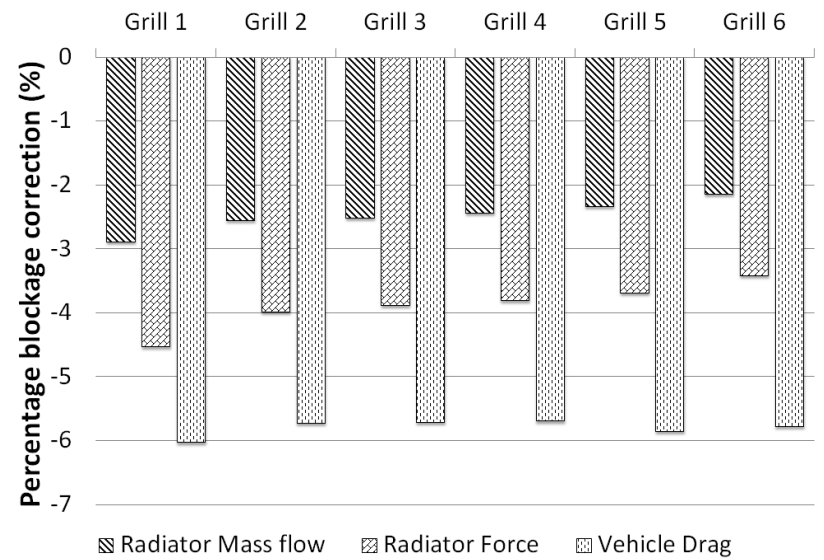

Figure 5. Percentage blockage correction needed to get equivalent of virtual tunnel values to open road.

\section{Results and Discussion}

\section{Wind-tunnel blockage effect}

Wind tunnels are a tool used by aerodynamicists, in order to quantify and improve the different aerodynamic properties of a vehicle. As it strives to get as close as possible to on road conditions, the result is a complex environment which introduces different errors, in the forms of blockage effects and measurement uncertainties, which need to be accounted for. In general the aerodynamic forces measured in the wind tunnel are an over prediction to the equivalent on road conditions. For example the drag force measured in the wind tunnel throughout these tests requires a correction of about $6 \%$ in order to represent the on road conditions. This raises concerns about the type of corrections that need to be applied to force measurements on the radiator core, and if the force on the core is over predicted due to the blockage effects then so is the mass flow through the radiator and therefore a correction is needed there as well. This has been investigated using CFD simulations where the car has been simulated in a virtual model of the Volvo wind tunnel and compared to open road simulations for the six different grill configurations. The numerical results of mass flow change, radiator core force change, and drag coefficient change can be seen in percentages in Figure 5. Distribution wise, no major changes could be seen apart from a general increase in the velocity magnitudes around the high velocity areas, an example is shown on Grill 3 in Figure 6. Figure 5 shows that on 


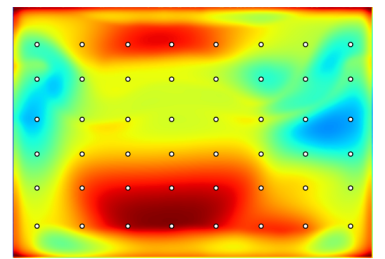

(a) Grill 3: Open road

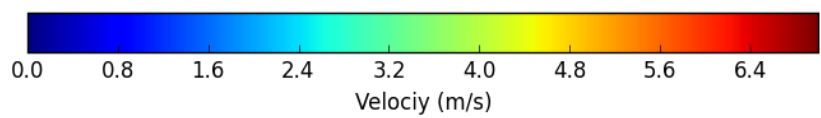

Figure 6. Comparison of velocity distribution for Grill 3 when simulated on open road or in virtual wind tunnel.

road conditions have around $6 \%$ lower overall $\mathrm{C}_{\mathrm{d}}$ which matches well with the correction applied in the wind tunnel tests, however it could also be seen that the correction needed for the force acting on the radiator core is not of the same magnitude, more importantly it varies with a constant drop as the blockage increases. A similar consistent trend is also observed in the mass flow of the radiator which further confirms the need for a correction. However, as finding such a correction is difficult, an alternative approach has been adopted where all comparisons of mass flow and forces acting on the radiator are performed between tests and simulations in a virtual tunnel, instead of open road. Although this potentially introduces additional errors to the simulations as the modelling of the tunnel in CFD is not necessarily accurate, the changes on the overall vehicle forces due to the presence of the tunnel are quite similar to those resulting from the test corrections and thus the blockage effect introduced in CFD is considered representative of the physical tunnels blockage effect.

\section{Effect of the 48 probe measurement grid}

In the wind tunnel tests, a measurement grid of 48 probes is used thus resulting in 48 local velocities. These points need to be interpolated/extrapolated over the radiator face in order to plot the distribution and to calculate the mass flow through the radiator. In the CFD simulations however, the velocity distributions and mass flows presented are the result of a significantly finer grid with a few hundred thousand points. The 48 probe grid distorts the distribution and the definitions while also missing out on most of the

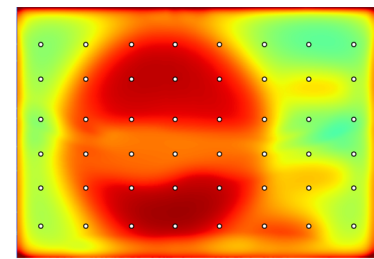

(a) Grill 1: CFD plot

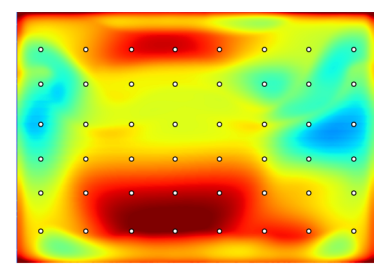

(c) Grill 3: CFD plot

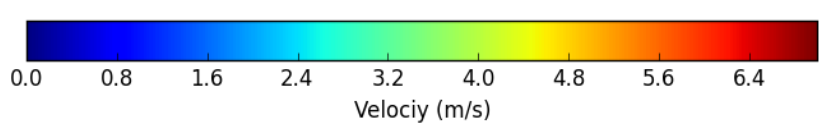

Figure 7. Comparison of numerical velocity distribution for two different grills when plotted directly from CFD vs plotted using only 48 measurement points similarly to experiments.

velocities outside the grid area. Thus for a more accurate comparison, the local velocities at the location of the 48 probes are extracted from the CFD simulations and then the distribution is plotted in an identical manner to that used in the wind tunnel tests. A further motivation can be seen in Figure 7 which compares the velocity distribution over two grills between continuous normal CFD plots and discrete 48 probe plots. The qualitative distribution varies and a deviation of quantitative mass flow results is introduced with an under prediction of $1.6 \%$ for Grill 1 and an over prediction of $2.0 \%$ for Grill 3. Also note that with the interpolation, larger errors in quantifying the effects of configuration changes are introduced. For example, a CFD comparison between Grill 1 and 3 shows a $6.3 \%$ decrease in mass flow, yet using the 48 probe approach the equivalent reduction is $9.6 \%$.

\section{Mass flow and force predictions}

Given the effects discussed, six different grill configurations have been tested in CFD on open road conditions and in a virtual tunnel. The results of the radiator core force difference to experiments on the radiator core are presented in Figure 8. The uncertainty of the load cell measurements 


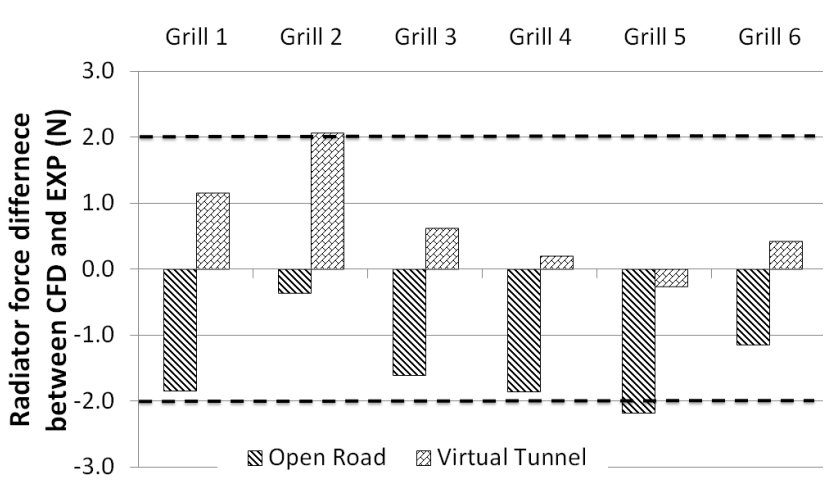

Figure 8. Delta in force prediction of the radiator core force between CFD and experiments. The dotted line outlines the uncertainty of the force measurement in experiments.

is $\pm 2 \mathrm{~N}$, thus the forces computed in CFD are mostly within the uncertainty of the measurements, with a slight over prediction for Grill 2. It could also be seen that the force acting on the radiator increases between open road and virtual tunnel. Figure 9 presents the mass flow comparison to experiments, while extracting mass flows from CFD using both full CFD integration as well as the 48 probe approach as it is believed to be more representative of the experimental setup. Although the initial error estimate of the pressure probes is believed to be within $\pm 5 \%$ when using 48 probes, the results reflect far larger deviations as the blockage over the grill increases up to the extreme condition where the grill is completely closed. It is interesting to note that looking only at the difference from open road simulations the delta between CFD and experiments is within the 5\% margin, except for Grill 6 which is clearly off. Figure 9 shows how using the 48 probe approach influences the mass flow differently for different configurations, while a clear trend of mass flow increase can be seen between open road to virtual tunnel which agrees well with the increase in forces. With a good match in forces between CFD and experiments, a significant mismatch in mass flow was not to be expected and thus a closer look into the distribution is performed based on Figure 10 comparing the virtual tunnel simulations and experiments. There is quite a clear qualitative difference in distribution between the CFD simulations and experimental results however improvements are detected when plotted using the 48 probe approach as this is what the experimental values are based on as well. However, low velocities below

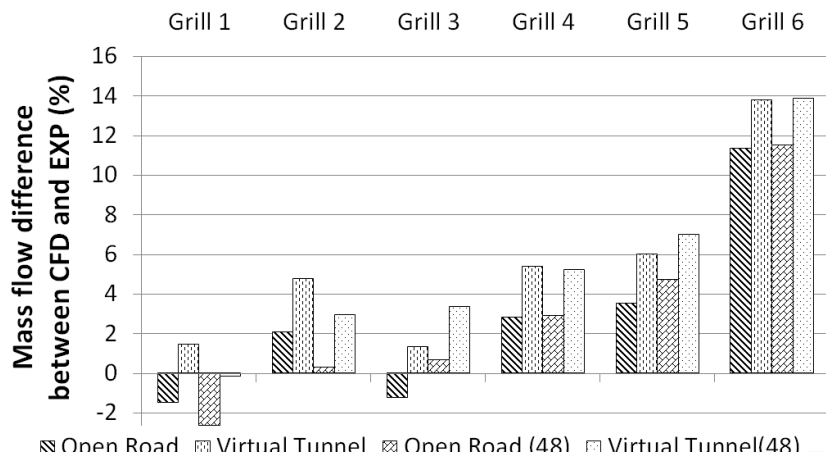

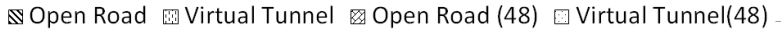

Figure 9. Delta in mass flow prediction through the radiator between CFD and experiments. The results are shown using direct CFD prediction and prediction using 48 virtual point measurements at identical positions to the probes in the experiments.

$4.5 \mathrm{~m} / \mathrm{s}$ seem to cause the largest error as they seem to be lower in the experiments than the CFD predictions. These can be seen behind the crash beam and the different grill blockages as low velocity areas in the experiments and although they are reasonably low velocities in CFD as well they are off by 1 to $2 \mathrm{~m} / \mathrm{s}$ thus resulting in significant mass flow errors when integrated over the area they cover. Furthermore, using the velocity distribution from pressure measurements, the theoretical force acting on the radiator can be calculated ${ }^{13}$. A clear mismatch between the theoretical and measured force values is detected with the pressure probes under predicting the force by $0.6,4.8$, 1.6, 7.9, 5.4, and 14.6\%, from Grill 1 through 6 respectively. Even though these figures cannot be directly compared to the mass flow percentage differences, presented in Figure 9 , as the force and mass flow are not linearly proportional, it does give an indication of a large discrepancy between the measured velocity distribution and the measured forces. A possible explanation to this could be that the probes lose resolution at high angles of oncoming flow, for example downstream of large blockage areas like a crash beam or a fully closed grill where pitch angles of above 65 degrees could be seen in the CFD simulations. This would likely affect the pressure readings as a probes calibration curve is obtained in ideal stable conditions in a test rig which naturally does not take into account high turbulence and separations at the inlets of the probes. 


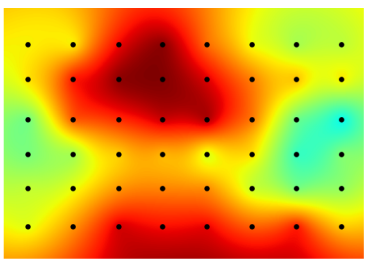

(a) Grill 1: Experiment

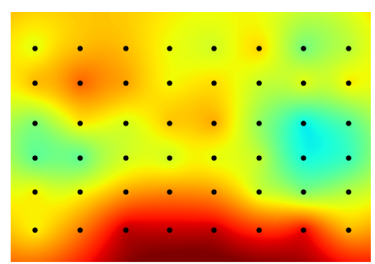

(c) Grill 2: Experiment

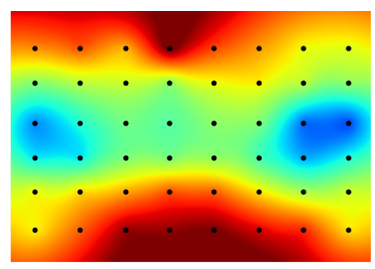

(e) Grill 3: Experiment

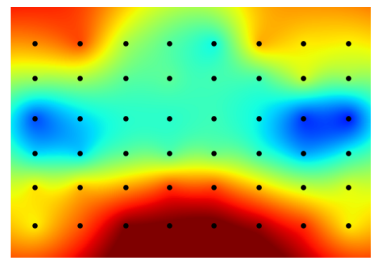

(g) Grill 4: Experiment

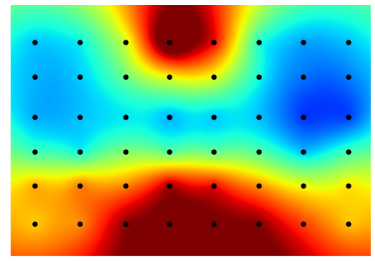

(i) Grill 5: Experiment

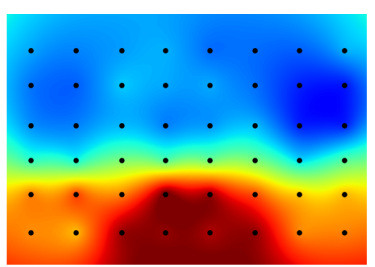

(k) Grill 6: Experiment

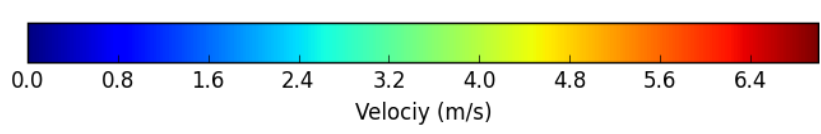

Figure 10. Velocity distribution over the radiator for the six different grill configurations from experiments and virtual tunnel CFD simulations, plotted using 48 virtual probe grid.

\section{Effect of wheels, moving ground, and mesh resolution}

Grill configurations have shown clear effects on cooling flow and cooling drag, however this is to be expected given the large blockage they impose at such close proximity upstream of the radiator. The outlets for cooling flow on the other hand are not clearly defined. After the flow passes through the fan shroud it exits in three major locations: the tunnel at the center of the car where exhaust pipe is located, or the two front wheelhouses. Rim designs and moving ground systems are known to have significant effects on the vehicles' overall drag and cooling drag by altering the underbody flow. This has been investigated by running the following configuration: fully covered rims, stationary front wheels, stationary rear wheels, and fully stationary five belt system. All of these configurations had significant changes on the overall vehicle drag yet only small changes, within uncertainty limits, could be detected on cooling flow, radiator velocity distribution, and the force acting on the radiator core. Thus it is believed that, for the tested vehicle, these configurations affect the interference drag at the cooling flow outlets yet have little to no effect on the flow inside the engine bay.

In agreement with the experimental results, simulations with fully closed rims showed very small effects on the cooling flow and radiator forces. However the position of the fan shroud had a large effect where moving the fan shroud $10 \mathrm{~mm}$ downstream changes the flow through the radiator by $10 \%$, roughly corresponding to $1 \%$ per mm. Also a mesh dependency study has been performed where a coarser and finer overall vehicle mesh with a max surface cell size of $2.5 \mathrm{~mm}$ have been analyzed with the changes in cooling flow not exceeding $2 \%$ between the coarsest and finest resolution, thus showing mesh independent results for the cooling flow and radiator force quantification. A minor shift of around 2 drag count and 5 to 10 lift count in the overall vehicle forces was observed which is attributed to a minor mesh dependency of the results.

\section{Effects on vehicle forces}

The cooling drag prediction for CFD simulations and experiments are presented in Figure 11. Note that the open 
road CFD simulations are to be compared with the corrected windtunnel results while the virtual tunnel simulations are to be compared with uncorrected windtunnel results thus eliminating the effects of the corrections used in the tunnel. The open road simulation results under predict cooling drag in most cases by about 5 count, however the results improve significantly when a virtual tunnel is used where the cooling drag predictions lie within 1.5 count which is within the uncertainty margins of both experiments and simulations. Even though the front lift changes are quite extreme as

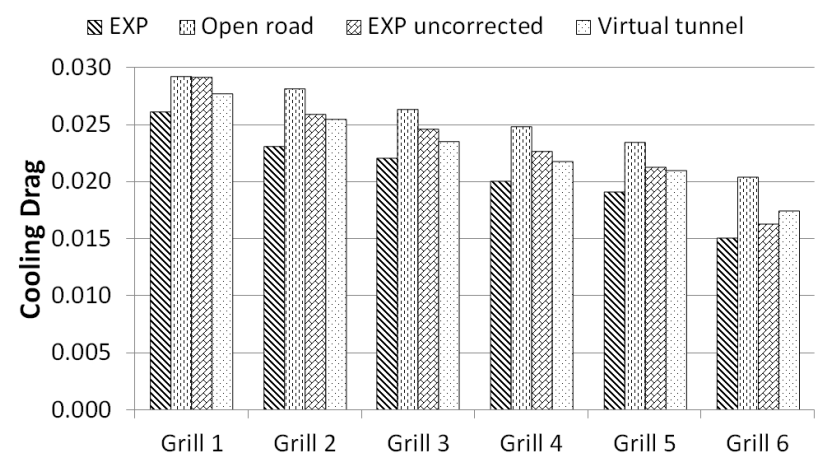

Figure 11. Cooling drag comparison between experiments and CFD. For a representative comparison, the experimental cooling drag figures are presented with and without tunnel blockage correction as the virtual tunnel CFD simulations should be compared to the uncorrected tunnel results.

shown in Figure 12, the simulation results seem to predict the results significantly better than with the virtual tunnel where the largest over prediction for Grill 1 is reduced from 16 to 8 count. However for a change of 114 count, an over prediction of 8 count is still acceptable.

The changes in rear lift are of smaller magnitude, as shown

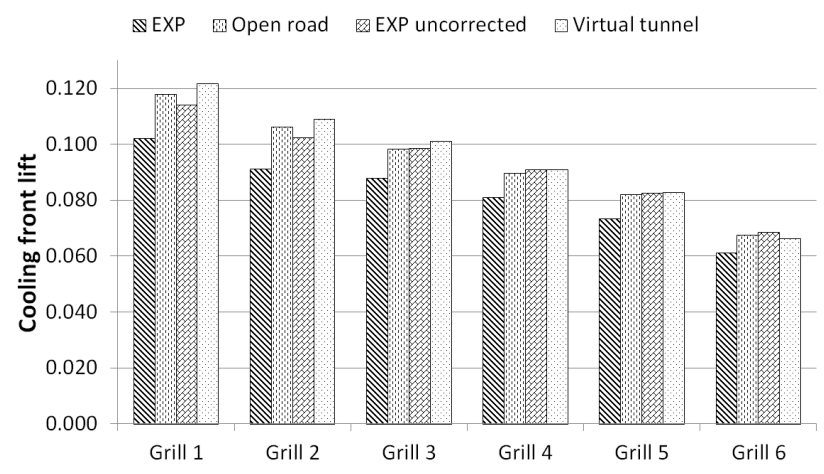

Figure 12. Cooling front lift comparison between experiments and CFD.

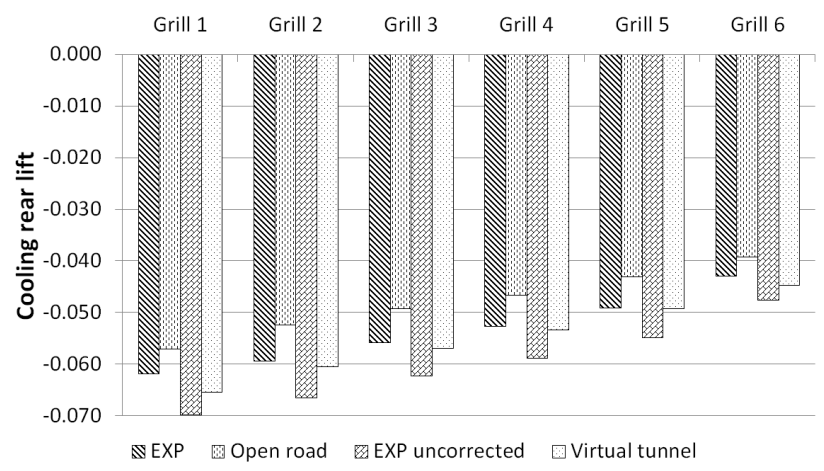

Figure 13. Cooling rear lift comparison between experiments and CFD.

in Figure 13, However the virtual tunnel did not seem to improve the predictions as the results seem to be a constant under prediction. This under prediction is of the order of 5 count which is considered within the uncertainty of the tunnel measurements and CFD simulations.

\section{Cooling drag vs radiator drag}

Given that the numerical results for force prediction acting on the radiator and overall cooling drag results matched well, the following section is solely based on experimental results. After the force acting on the radiator core is measured, a drag contribution can be estimated, this is reported as radiator drag in Figure 14. The cooling drag for each of the configurations is also presented and in comparison the cooling drag is roughly three times lower than the drag force acting on the radiator. However Garrone $^{6}$ reports a cooling drag more than five times lower than the cooling package drop, yet his configuration included ducted outlet into the wheel houses which have low pressure, thus significantly reducing the interference drag and possibly even having negative interference.

\section{Conclusion}

In summary, an investigation of cooling flow quantification has been performed both numerically and experimentally. A particular effect investigated in CFD simulations has been that of wind tunnel blockage which showed significant effects on the results. Although this cannot be generalized to all wind tunnels, it is an effect worth investigating when looking at accurate comparison between CFD and tests. 


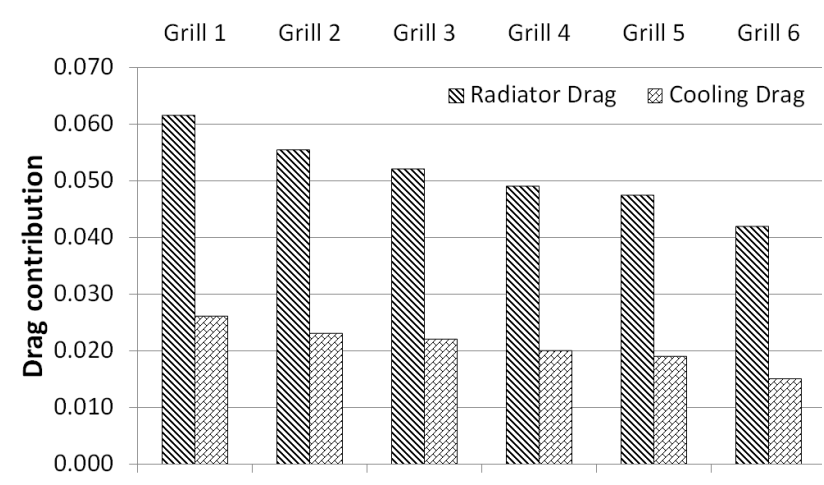

Figure 14. Contribution of radiator force to drag in comparison with cooling drag.

Also for validation purposes, a more detailed analysis of the accuracy of pressure probes is required as a significant discrepancy in experimental results could be seen between probe and force measurements when the blockage imposes a highly non-uniform distribution through the radiator and high pitch angles. Although the forces seem to match quite well with CFD predictions, it is believed that the probes are under predicting the flow through the radiator when located in the wake of upstream blockages. In the case of a more complete cooling package with a condenser upstream of the radiator, this could act as a flow straightener and enable the pressure probes to give more accurate measurements, yet this remains to be investigated in future work.

It has also been shown that using virtual measurement points in CFD results in a more comparable qualitative distribution when validating to experiments even though it could offset the results significantly. Finally, including the geometry of the physical wind tunnel in CFD has shown to improve the prediction of cooling drag and lift distribution changes to a level where the error lies within the uncertainty of the tests and simulations.

Although the cooling package in this work is relatively simplified, the baseline changes in cooling drag and lift are very similar to tests performed with a complete cooling package thus similar trends are expected.

\section{Acknowledgements}

The authors would like to thank Volvo Cars and Volvo Trucks for providing access to their testing facilities. Special thanks to Dr Peter Gullberg at Volvo Trucks for his help with calibration and Dr Christoffer Landström for support during the wind tunnel tests and discussion of results.

\section{Funding}

Energimyndigheten (Swedish Energy Agency) project number 37195-1.

\section{References}

1. Hucho WH. Aerodynamics of Road Vehicles. Fourth ed. Warrendale, USA: Society of Automotive Engineers, Inc, 1998. ISBN 0-7680-0029-7.

2. Tesch G, Demuth $\mathrm{R}$ and Adams N. A new approach to analyzing cooling and interference drag. SAE Int $J$ Passeng Cars Mech Syst 2010; 3: 339-351. DOI:10.4271/ 2010-01-0286. URL http://dx.doi.org/10.4271/ $2010-01-0286$.

3. Williams J. Aerodynamic drag of engine-cooling airflow with external interference. In SAE Technical Paper. SAE International. DOI:10.4271/2003-01-0996. URL http: //dx.doi.org/10.4271/2003-01-0996.

4. Wolf T. Minimising the cooling system drag for the new porsche 911 carrera. In 5th MIRA International Conference of Vehicle Aerodynamics. Warwick, UK.

5. Soja $\mathrm{H}$ and Wiedemann J. The interference between exterior and interior flow on road vehicles. Journee d'etude: Dynamique du vehicule Securite Active 1987; : 101-105.

6. Garrone A and Masoero M. Car underside, upperbody and engine cooling system interactions-and their contributions to aerodynamic drag. In SAE Technical Paper. SAE International. DOI:10.4271/860212. URL http://dx . doi.org/10.4271/860212.

7. Williams J, Karanth D and Oler W. Cooling inlet aerodynamic performance and system resistance. In SAE Technical Paper. SAE International. DOI:10.4271/2002-01-0256. URL http://dx.doi.org/10.4271/2002-01-0256.

8. Kuthada $\mathrm{T}$ and Wiedemann $\mathrm{J}$. Investigations in a cooling air flow system under the influence of road simulation. In SAE Technical Paper. SAE International. DOI:10.4271/ 2008-01-0796. URL http://dx.doi.org/10.4271/ 2008-01-0796.

9. Yang Z, Nastov A and Schenkel M. Further assessment of closed-wall wind tunnel blockage using cfd. In SAE Technical 
Paper. SAE International. DOI:10.4271/2005-01-0868. URL http://dx.doi.org/10.4271/2005-01-0868.

10. Kuthada T. A review of some cooling air flow measurement techniques for model scale, full scale and cfd. SAE Int J Passeng Cars - Mech Syst 2013; 6: 88-96. DOI:10.4271/ 2013-01-0598. URL http://dx.doi.org/10.4271/ 2013-01-0598.

11. Sterneus J, Walker T and Bender T. Upgrade of the volvo cars aerodynamic wind tunnel. In SAE Technical Paper. Detroit, Michigan: SAE International. DOI:10.4271/2007-01-1043.

12. Ruijsink R. The use of the microprobe system in cooling system development. In Third MIRA International Vehicle Aerodynamics Conference. Rugby, UK. ISBN 0952415623.

13. Hobeika T. On Tyre Rotation Modelling and Cooling Flow Measurements. Institutionen för tillmpad mekanik, Fordonsteknik och autonoma system, Chalmers tekniska högskola,, 2015.

14. Tropea C, Yarin A and Foss J. Springer Handbook of Experimental Fluid Mechancis. Heidelberg, Germany: Springer, 2007. ISBN 978-3-540-25141-5.

15. Wall A. Simulating the Volvo Cars Aerodynamic Wind Tunnel with CFD. Diploma work - Department of Applied Mechanics, Chalmers University of Technology, Göteborg, Sweden, no: 2013:08, Institutionen för tillmpad mekanik, Fordonsteknik och autonoma system, Chalmers tekniska högskola,, 2013. 